\title{
Application of Markov Model in Human Resource Supply Forecasting in Enterprises
}

\author{
Wan-yin Du, Shou Li \\ Department of Information Management, The Central Institute For Correctional Police, \\ Baoding,071000, China
}

Keywords: Human resource supply; Gray theory; Markov model; Forecasting model

\begin{abstract}
A complete human resources strategic planning can become the core competitiveness of enterprises, which is an important means to achieve competitive advantage. This paper focused on improved gray Markov model in human resource internal supply forecasting, so that enterprises can reasonably predict their internal human resource supply through this method and provide an important guarantee for enterprises to develop human resources strategic planning.
\end{abstract}

\section{Introduction}

Human resources strategic planning is the foundation of human resource management. The scientific human resources strategic planning is related to future growth and strategic objectives of the enterprise. In essence, human resource planning is to ensure that a business can be obtained in a timely manner and apply the right amount of personnel procedures. Through this program, the human resources can be used economically and effectively [1-3]. A comprehensive and rational human resource strategic planning usually involves three aspects: human resource demand and supply forecasts, dynamic balance demand and supply of human resources, incentives embodied in human resources strategic planning. Human resource planning is the term used to describe how companies ensure that their staffs are the right staff to do the jobs. Sub topics include planning for staff retention, planning for candidate search, training and skills analysis and much more. Rigorous HR planning links people management to the organization's mission, vision, goals and objectives, as well as its strategic plan and budgetary resources. A key goal of HR planning is to get the right number of people with the right skills, experience and competencies in the right jobs at the right time at the right cost $[4,5]$.

Without scientific and quantitative forecasting analysis on internal supply and external supply of enterprises human resources, enterprises in China only rely on manager personal experience in human resource supply forecasting problem. As a result, wrong decision of human resources enterprises, improper expansion of staff size and staff structural imbalance emerge. How to make forecasting analysis on internal supply and external supply of human resources in enterprises and ensure human resource supply for enterprises is a problem to be solved urgently[6,7].

When corporate human resource supply forecasting, we often encounter such difficulties, the changing internal and external environment, decision-makers can not get accurate and timely information. It is difficult to accurately predict the actual situation of human resource supply[8,9].

As Peter Drucker emphasized that human resource planning must be based on changes in the environment, from identifying, analyzing major contradictions and problems to achieve corporate strategic human resources facing the starting level. The Improved gray Markov model embodies the advantages of this area, it is an effective method of internal human resource supply forecasting solution[10].

\section{The selection method on internal supply forecasting of human resources}

Common methods on internal supply forecasting of human resources contain staff skills list, staff replacement model, Markov model.

Staff skills list contains education, training, work experience, qualifications, ability level, the competent evaluation, etc. Staff skills list makes it easy to estimate the possibility of changing 
guard for assigned staff and forecast the internal supply of human resources. However, the method of staff skills list requires the enterprises must build staff skills list database, timely maintenance and update it to reflect the latest status of enterprises human resources.

Staff replacement model is a simple method aiming at internal supply forecasting of the key staff positions and management positions. It is widely used both in large and small enterprises. This method marks incumbents and potential

substitutes of major positions in enterprises through staff replacement planning diagram and then make the staff replacement plan.

As an internal supply forecasting method of enterprises human resources, Markov model is a kind of transition probability matrix. Through statistical analysis of historical data, it finds the rule of personnel transfer, fully forecasts the probability and the number of internal personnel transfer, and then forecast the internal supply of enterprises human resources. The basic assumption of this model is that the enterprises staff transfer is regular and the transition probability is subject to certain distribution.

According to analysis and comparison of these forecasting methods, Markov model not only considers the staff promotion prospects, directly produces promotion policies in the forecasting process, but also can solve the problems of human resources internal supply forecast in the large enterprises with complex staff category.

\section{The gray GM(1,1) model}

Features of gray GM $(1,1)$ model are gray prediction, singular columns prediction, and first-order linear model with a single variable. It sets up model under the use of time series arrangement, and analyzes the data regularity. Gray system is affected by many factors and cannot determine the amount of complex relationships which called gray amount. In order to predict the gray amount, the model looks for useful information from its own time series model, and discoveres and understands of the internal law.

GM $(1,1)$ model accumulates the original sequence $x^{(0)}(1), x^{(0)}(2), \cdots, x^{(0)}(n)$ to obtain new data sequence. Among it,

$$
x^{(1)}(j)=\sum_{i=1}^{j} x^{(0)}(i) \quad j=1,2, \cdots, n .
$$

Establish GM $(1,1)$ model of first-order differential equations after data accumulation:

$$
\frac{d x^{(1)}(t)}{d t}+a x^{(1)}(t)=u
$$

According to differential equations, using the least squares method to obtain a differential equation:

$$
\bar{x}^{(1)}(t+1)=\left(\bar{x}^{(1)}(0)-\frac{u}{a}\right) e^{-a t}+\frac{u}{a} .
$$

Because the prediction equation is to accumulate data column, so a regressive reduction can be predicted value data columns.

$$
\bar{x}^{(0)}(t)=\left(\bar{x}^{(1)}(t+1)-\bar{x}^{(1)}(t)\right)
$$

\section{Improved gray GM $(1,1)$ model}

Improved algorithm of gray GM $(1,1)$ model is not the accumulation of original sequence, but to establish a first-order differential equations directly on the raw data series:

$$
\frac{d x(t)}{d t}+a x(t)=u
$$

Firstly, the Eq. (5) can be rewritten as: 


$$
\frac{d x(t)}{d t}=-a x(t)+u
$$

$t$ is replaced by $t+1$ with substitutes, and means with the original formula for the arithmetic.

Because of:

$$
\frac{1}{2}\left(x^{\prime}(t+1)+x^{\prime}(t)\right) \approx x^{\prime}\left(t+\frac{1}{2}\right)=x(t+1)-x(t)=\Delta x(t)
$$

We can obtain:

$$
a\left(-\frac{1}{2}(x(t+1)+x(t))\right)+u=\frac{1}{2}\left(x^{\prime}(t+1)+x^{\prime}(t)\right) \approx \Delta x(t)
$$

Set $A=[a, u]^{T}$, and estimate the coefficient matrix by least squares method.

$A=\left(B^{T} B\right)^{-1}\left(B^{T} C\right)$.

Among it,

$$
\begin{aligned}
& B=\left[\begin{array}{c}
-\frac{1}{2}(x(0)+x(1)) \\
-\frac{1}{2}(x(1)+x(2)) \\
\vdots \\
-\frac{1}{2}(x(n-1)+x(n))
\end{array}\right] . \\
& C=\left[\begin{array}{llll}
\Delta x(0) & \Delta x(1) & \cdots & \Delta x(n-1)
\end{array}\right] .
\end{aligned}
$$

Bring the value $a, \quad u$ in and build a first-order differential equations. Through transformation and integration, prediction function can be drawn:

$$
x(t)=\left(x(0)-\frac{u}{a}\right) e^{-a t}+\frac{u}{a} .
$$

\section{Markov process}

Markov process is a random process with no aftereffect nature. The so-called no aftereffect refers to: When the process is in a state in which the time $t_{m}$ is known, the probabilistic characteristics of the course of time $t$ greater than $t_{m}$ only has the relationship with the state of $t$, and has no relationship with the previous state [1,2].

Set the discrete state space of random sequence $\{X(n), n=1,2, \cdots\}$ as E. For non-negative integer $n_{1}, n_{2}, \cdots, n_{m}\left(0 \leq n_{1}<n_{2}<\cdots<n_{m}\right)$, and natural number $k$, and optionally $i_{1}, i_{2}, \cdots, i_{m}, j \in E$, satisfy:

$$
P\left\{X\left(n_{m}+k\right)=j \mid X\left(n_{1}\right)=i_{1}, X\left(n_{2}\right)=i_{2}, \cdots, X\left(n_{m}\right)=i_{m}\right\}=P\left\{X\left(n_{m}+k\right)=j \mid X\left(n_{m}\right)=i_{m}\right\}
$$

Thus call random sequence $\{X(n), n=1,2, \cdots\}$ as Markov process.

The probability of the condition on the right of Eq. (13):

$$
P\{X(n+k)=j \mid X(n)=i\}, k \geq 1
$$

Set $k$ step transition probability at time of $n$ in Markov chains, denote $p_{i j}(n, n+k)$.

The process of transition probabilities $i$ are known as the state at the time $n$, after a unit of time $k$, the process is in a state of probability $j$. The time Markov chains which transition probability does not depend on called time homogeneous markov chain[3].

Obviously, the transition probability matrix of all the elements are non-negative, and the elements of each row is equal to one. Easy to see, k-step transition probability matrix $P(k)$ has important relationship with one step transition probability matrix $P$. 


$$
P(k)=P^{k} \text {. }
$$

Another important property of the relevant transition probabilities of the Markov chain is ergodic of Markov chain. The so-called ergodic means: if the limit Markov chain transition probabilities of existence, and has nothing to do with i, namely:

$$
\lim _{k \rightarrow \infty} p_{i j}(k), i, j \in E \text {. }
$$

It is easy to show that for finite Markov chain, if there is a positive integer $\mathrm{K}$,

$p_{i j}(k)>0, i, j=1,2, \cdots, N$.

Thus the chain is ergodic, and limit distribution $\left\{p_{j}, j=1,2, \cdots, N\right\}$ is the unique solution.

$$
p_{j}=\sum_{i=1}^{N} p_{i} p_{i j}, j=1,2, \cdots, N \text {. }
$$

Which satisfy the conditions:

$$
p_{j}>0 \quad(j=1,2, \cdots, N), \sum_{j=1}^{N} p_{j}=1 \text {. }
$$

\section{The improve gray Markov Model}

The improved algorithm concrete steps are shown as follows [4,5]:

(1) According $\operatorname{GM}(1,1)$, the forecasting function is obtained.

$X(t)=\left[x(0)-\frac{u}{a}\right] e^{-a t}+\frac{u}{a}$.

(2) According to the forecasting curve, combined with the specific values in each year, the state sections are obtained through divided, which parallel to the prediction function. Each state is the interval containing the next free.

$$
\otimes_{i}=\left[\otimes_{1 i}, \otimes_{2 i}\right] \text {. }
$$

Where $\otimes_{1 i}=x(t)+A_{i}, \otimes_{1 i}=x(t)+B_{i}, A_{i}=a_{i} y, B_{i}=b_{i} y$.

(3) Calculate the state transition probability matrix. Its calculation equation is shown as follows:

$$
P_{i j}=\frac{n_{i j}(k)}{n_{i}} \text {. }
$$

The transition number from state $E_{i}$ to state $E_{j}$ is $n_{i j}(k)$. The occurrence number of state $E_{i}$ is $n_{i}$. Then, the state transition probability matrix is obtained.

$$
P_{k}=\left[\begin{array}{ccccc}
P_{11 k} & \cdots & P_{12 k} & \cdots & P_{1 m k} \\
P_{21 k} & \cdots & P_{22 k} & \cdots & P_{2 m k} \\
\vdots & \vdots & \vdots & \vdots & \vdots \\
P_{m 1 k} & \cdots & P_{m 2 k} & \cdots & P_{m m k}
\end{array}\right] .
$$

State transition matrix reflects the transfer rule within the system between each state by state transition probability matrix and the initial state. It is possible to determine the future trends.

(4) Calculate the forecasting value. According to the obtained data and the state transition probability matrix, the next transition state can be determined, and the change interval of the forecasting value $\left[\otimes_{1 i}, \otimes_{2 i}\right]$ is also determined. The forecasting value is defined as the the midpoint of the interval.

$$
y=x(t)+\frac{A_{i}+B_{i}}{2} .
$$




\section{Specific example and results analysis}

The human resource supply data of a certain power plant is used in this paper. The total number of workers in the proposed power plant in 2012 is 1264, which can be divided into three categories: managers, operators and maintenance personnel. The numbers of these three kind of worker are respectively 232, 462 and 580. The total number of workers in the proposed power plant in 2013 is 1248. The numbers of managers, operators and maintenance personnel are respectively 230,454 and 564.

According to the above data, the personnel transition probability matrix is constructed.

$$
P=\left[\begin{array}{ccc}
0.94 & 0.04 & 0 \\
0.01 & 0.95 & 0.02 \\
0 & 0.03 & 0.95
\end{array}\right]
$$

The data in 2012 is regarded as the initial value. According to the staff transition probability matrix, predict the number of companies supply in the nest years.

Through the calculation of worker in the three years 2010,2011,2012, the personnel flow between the various levels inside the plant is stable. Human resources obtained from outside the plant comes mainly from the following two aspects: firstly, fresh college graduates of all colleges and universities; Secondly, demobilized military personnel have been regular professional training and learning. According to the last three years the company reported to the actual number, the plant is about to get total 20 personnel from outside in each year, which include three managers, seven operators and 10 maintenance personnel, namely:

$$
R(t)=\left[\begin{array}{lll}
r_{1}(t) & r_{2}(t) & r_{3}(t)
\end{array}\right]=\left[\begin{array}{lll}
3 & 7 & 10
\end{array}\right] .
$$

The human resource supply situation in the next few years can be calculated accordingly.

$$
\begin{aligned}
& N_{2013}=\left[\begin{array}{lll}
226 & 459 & 570
\end{array}\right] . \\
& N_{2014}=\left[\begin{array}{lll}
220 & 455 & 561
\end{array}\right] . \\
& N_{2015}=\left[\begin{array}{lll}
214 & 451 & 552
\end{array}\right] .
\end{aligned}
$$

From the above results data, Table 1 is obtained.

Table 1: Human resource supply forecasting results

\begin{tabular}{cccc}
\hline Year & Manager & Operator & $\begin{array}{c}\text { Maintenance } \\
\text { personnel }\end{array}$ \\
\hline 2013 & 226 & 459 & 570 \\
2014 & 220 & 455 & 561 \\
2015 & 214 & 451 & 552 \\
\hline
\end{tabular}

Compare with the actual data in 2013, the relative error of three kind of worker are respectively $1.74 \%, 1.1 \%$ and $1.06 \%$, which are all low. The result can demonstrate that the improved gray Markov model can be feasibly applied in human resource supply forecasting.

\section{Conclusions}

Through the establishment of the improved Markov process, the human resource supply is predicted. The amount of personnel supply after many times transition is determined by the way of personnel transferring. This proposed method can provide guidance for business managers and human resource professionals. 


\section{References}

[1] Hara Charalambous, Apostolos Thoma, Marius Vladoiu. Markov complexity of monomial curves. Journal of Algebra. 417 (5), pp. 391-411,2014.

[2] Ting-Li Chen, Chii-Ruey Hwang. Accelerating reversible Markov chains. Statistics \& Probability Letters. 83 (6), pp. 1956-1962.,2013.

[3] Francesco Altomare, Mirella Cappelletti Montano, Vita Leonessa, Ioan Raşa. On Markov operators preserving polynomials. Journal of Mathematical Analysis and Applications. 415 (11), pp. 477-495,2014.

[4] John van der Hoek, Robert J. Elliott. A modified hidden Markov model. Automatica. 49 (5), pp. 3509-3519,2013.

[5] Alessio Farcomeni. Hidden Markov partition models. Statistics \& Probability Letters. 81(7), pp. 1766-1770,2011.

[6] A. Fort, M. Mugnaini, V. Vignoli. Hidden Markov Models approach used for life parameters estimations. Reliability Engineering \& System Safety. 136, pp. 85-91, 2015.

[7] Zhaoning Gong, Tianxiang Cui, Ruiliang Pu, Chuan Lin, Yuzhu Chen. Dynamic simulation of vegetation abundance in a reservoir riparian zone using a sub-pixel Markov model. International Journal of Applied Earth Observation and Geoinformation. 35, pp.175-186, 2015.

[8] Mario Hellmich, Heinz-Peter Berg. Markov analysis of redundant standby safety systems under periodic surveillance testing. Reliability Engineering \& System Safety, 133, pp. 48-58, 2015.

[9] S. Akshay, Timos Antonopoulos, Joël Ouaknine, James Worrell. Reachability problems for Markov chains. Information Processing Letters. 115(2), pp. 155-158, 2015.

[10] R. Faddoul, W. Raphael, A.-H. Soubra, A. Chateauneuf. Partially Observable Markov Decision Processes incorporating epistemic uncertainties. European Journal of Operational Research, 241(2), pp. 391-401, 2015. 